\title{
A TABLE OF MULTIPLY PERFECT NUMBERS.
}

\author{
BY PROFESSOR R. D. CARMICHAEL.
}

(Kead before the American Mathematical Society, February 23, 1907.)

A MULTIPLy perfect number is one which is an exact divisor of the sum of all its divisors, the quotient being the multiplicity.* The object of this paper is to exhibit a method for determining all such numbers up to $1,000,000,000$ and to give a complete table of them. I include an additional table giving such other numbers as are known to me to be multiply perfect.

Let the number $N$, of multiplicity $m(m>1)$, be of the form

$$
N=p_{1}^{a_{1}} p_{2}^{a_{2}} \cdots p_{n}^{\alpha_{n}}
$$

where $p_{1}, p_{2}, \cdots, p_{n}$ are different primes. Then by definition and by the formula for the sum of the factors of a number, we have

(1) $m=\frac{p_{1}^{a_{1}}+p_{1}^{a_{1}-1}+\cdots+p_{1}+1}{p_{1}^{a_{1}}} \cdots \frac{p_{n}^{a_{n}}+p_{n}^{a_{n}-1}+\cdots+p_{n}+1}{p_{n}^{a_{n}}}$.

Hence

$$
m<\frac{p_{1}}{p_{1}-1} \cdot \frac{p_{2}}{p_{2}-1} \cdots \frac{p_{n}}{p_{n}-1} .
$$

These formulas will be of frequent use throughout the paper.

Since $2 \cdot 3 \cdot 5 \cdot 7 \cdot 11 \cdot 13 \cdot 17 \cdot 19 \cdot 23=223,092,870$, multiply perfect numbers less than $1,000,000,000$ contain not more than nine different prime factors; such numbers, lacking the factor 2 , contain not more than eight different primes; and, lacking the factors 2 and 3, they contain not more than seven different primes.

First consider the case in which $N$ does not contain either 2 or 3 as a factor. By equation (2) we have

$$
m<\frac{5}{4} \cdot \frac{7}{6} \cdot \frac{11}{10} \cdot \frac{13}{12} \cdot \frac{17}{16} \cdot \frac{19}{1} \frac{2}{8} \cdot \frac{23}{2}=\frac{6}{3} \frac{7}{3} \frac{6}{17} \frac{3}{7} \frac{9}{6} .
$$

Hence $m=2$; moreover seven primes are necessary to this value. Now, $5 \cdot 7 \cdot 11 \cdot 13 \cdot 17 \cdot 19 \cdot 23=37,182,145$. Therefore, since we are not to consider numbers greater than $1,000,000,000$,

* The name " multiply perfect" was introduced by Lehmer, Annals of Math., ser. 2, vol. 2, p. 103 . 
not more than one of the seven prime factors of $N$ can occur to a power higher than the first. Then every numerator except one in the second member of equation (1) will contain the factor 2, while the denominators are all odd. Therefore, since $m$ now equals 2 , it is clearly impossible to satisfy equation (1).

Next consider the case in which $N$ contains the factor 3 but not the factor 2. By (2) we may show that $m=2$ or 3 . The value $m=3$ is readily excluded by the method of the last paragraph. Let us examine the case $m=2$. With the present values equation (1) becomes

$$
\begin{gathered}
2=\frac{3^{a_{1}}+\cdots+3+1}{3^{a_{1}}} \cdot \frac{p_{2}^{a_{2}}+\cdots+p_{2}+1}{p_{2}^{a_{2}}} \cdots \\
\frac{p_{n}^{a_{n}}+\cdots+p_{n}+1}{p_{n}^{a_{n}}} .
\end{gathered}
$$

Since each denominator of (4) is odd the prime 2 can enter as a factor only once in the numerator of the second member. Therefore $a_{1}$ is even. If $a_{1}>8$ it is easy to show that $N$ is greater than $1,000,000,000$. The primes introduced when $a_{1}=8$ or $a_{1}=6$ in each case enable us to exclude these values with readiness. We have left then to consider only the values $a_{1}=4,2$. Our work will be abridged as much as possible.

(1) If $a_{1}=4$, we have $p_{2}=11$. It is then easy to show that $a_{2}=2$ or 4 . (a) If $a_{2}=2$, we have readily $p_{3}=7$, $p_{4}=19, a_{3} \geqq 2, a_{4}=2, p_{5}=127$. (b) If $a_{2}=4$, we have $p_{3}=5, p_{4}=3221, a_{4}=1, p_{5}=179, a_{5} \geqq 2$. Both cases exceed the limit.

(2) If $a_{1}=2$, we have $p_{2}=13$. The possible values of $a_{2}$ are $1,2,4$. (a) If $a_{2}=1$, then $p_{3}=7$ and $a_{3}=2$ or 4 . If $a_{3}=2$, we have $p_{4}=19, a_{4}=2, p_{5}=127, a_{5}=2, p_{6}=5419$. If $a_{3}=4, p_{4}=2801$ and $a_{4}>1$. Both cases exceed the limit. (b) If $a_{2}=2$, we have $p_{3}=61$ and $a_{3}=1,2,3$. The value $a_{3}=3$ is excluded by its bringing into the numerator of (4) too high a power of 2 . If $a_{3}=2$, we have $p_{4}=97, a_{4}=1$, $p_{5}=7$. If $a_{3}=1$, we have $p_{4}=31, a_{4}=2, p_{5}=331$. Both cases again exceed the limit. (c) If $a_{2}=4, p_{3}=30941$, which at once makes $N$ too great.

This completes the discussion. No perfect number has been found. Hence

There are no multiply perfect odd numbers $<1,000,000,000$.*

* There are no odd numbers known to be multiply perfect. See Encyklopädie der Mathematischen Wissenschaften, vol. $\mathrm{I}_{2}$ (IC1), p. 578. 
If the number $N$ is even, it is easy to show from (2) that $m \ngtr 6$, and that if $m=6$ nine primes are necessary. From the equation $2 \cdot 3 \cdot 5 \cdot 7 \cdot 11 \cdot 13 \cdot 17 \cdot 19 \cdot 23=223,092,870$ we readily see that when $N$ contains 9 different prime factors every such factor except 2 and 3 enters to a power not higher than the first and that 2 enters to a power not higher than the third. It is easy to show that these conditions do not satisfy (1); therefore $m \ngtr 5$.

Now $N$ cannot contain 2 to a power higher than the fifteenth without going beyond our assigned limit. By the method illustrated above for the case $N$ odd and with the aid of the preceding deductions, I have taken each value of $a_{1}$ from 1 to 15 ( $a_{1}$ being the exponent of 2 in the factors of $N$ ) and have thus determined all the multiply perfect numbers up to $1,000,000,-$ 000 . To insure accuracy $I$ have also computed these numbers by another method depending upon certain results which $\mathrm{I}$ have not yet published.

The first table below contains all the multiply perfect numbers up to $1,000,000,000$. The second table contains certain such numbers above $1,000,000,000$. These two tables embrace all the numbers known to the writer to be multiply perfect. According to Mersenne $2^{127}-1$ and $2^{257}-1$ are prime; but since this result is uncertain I do not include in the table $2^{126}\left(2^{127}-1\right)$ and $2^{256}\left(2^{257}-1\right)$ which are perfect if Mersenne is correct.

\section{Table of all Multiply Perfect Number}

BeLOW 1,000,000,000.

\begin{tabular}{rc|cc} 
Number. & Multiplicity. & Number. & Multiplicity. \\
6 & 2 & 523776 & 3 \\
28 & 2 & 2178540 & 4 \\
120 & 3 & 23569921 & 4 \\
496 & 2 & 33550336 & 2 \\
672 & 3 & 45532800 & 4 \\
8128 & 2 & 142990848 & 4 \\
30240 & 4 & 919636480 & 3 \\
32760 & 4 & & \\
TABLE OF CERTAIN MULTIPLY PERFECT NUMBERS \\
\multicolumn{3}{c}{ ABOVE 1,000,000,000. } \\
\multicolumn{3}{c}{ Number. } \\
$2^{7} \cdot 3^{4} \cdot 5 \cdot 7 \cdot 11^{2} \cdot 17 \cdot 19$ & Multiplicity. \\
$2^{8} \cdot 3 \cdot 5 \cdot 7 \cdot 19 \cdot 37 \cdot 73$ & 4 \\
\multicolumn{3}{c}{} \\
\multicolumn{3}{c}{}
\end{tabular}


$2^{10} \cdot 3^{4} \cdot 5 \cdot 7 \cdot 11^{2} \cdot 19 \cdot 23 \cdot 89$

$2^{13} \cdot 3 \cdot 11 \cdot 43 \cdot 127 \quad 3$

$2^{14} \cdot 5 \cdot 7 \cdot 19 \cdot 31 \cdot 151 \quad 3$

$2^{14} \cdot 3 \cdot 5 \cdot 7 \cdot 19 \cdot 31 \cdot 151$

$2^{15} \cdot 3^{5} \cdot 5^{2} \cdot 7^{2} \cdot 11 \cdot 13 \cdot 17 \cdot 19 \cdot 31 \cdot 43 \cdot 257 \quad 6$

$2^{16} \cdot 131071 \quad 2$

$2^{18} \cdot 524287 \quad 2$

$2^{30} \cdot 2147483647 \quad 2$

$2^{60} \cdot 2305843009213693951 \quad 2$

Pregbyterian College, Anniston, Ala.,

February, 1907.

THE SYMMETRIC GROUP ON EIGHT LETTERS

AND THE SENARY FIRST HYPOABELIAN GROUP.

BY PROFESSOR L. E. DICKSON.

(Read before the Chicago Section of the American Mathematical Society, March 30, 1907.)

THE set of all transformations

$$
\xi_{i}^{\prime}=\sum_{j=1}^{3}\left(\alpha_{i j} \xi_{j}+\gamma_{i j} \eta_{j}\right), \quad \eta_{i}^{\prime}=\sum_{j=1}^{3}\left(\beta_{i j} \xi_{j}+\delta_{i j} \eta_{j}\right) \quad(i=1,2,3)
$$

with integral coefficients taken modulo 2 which leave invariant the quadratic form

$$
\xi_{1} \eta_{1}+\xi_{2} \eta_{2}+\xi_{3} \eta_{3}
$$

form a group $G_{0}$, called the (total) senary first hypoabelian group. It is a subgroup of the senary abelian linear group. The order of $G_{0}$ is (Linear Groups, page 206)

$$
2\left(2^{3}-1\right)\left(2^{4}-1\right) 2^{4}\left(2^{2}-1\right) 2^{2}=8 \text { ! }
$$

The object of this note is to prove that $G_{0}$ is simply isomorphic with the symmetric group on eight letters.

We make use of the subgroup $J_{0}$ of $G_{0}$ obtained as the second compound of the general quaternary linear homogeneous group $Q$ modulo 2 (Linear Groups, page 208). The process is analogous to the formation of the determinant of the sixth order, 